\title{
Partial information decomposition as a unified approach to the characterization and design of neural goal functions
}

\author{
Michael Wibral ${ }^{1,2^{*}}$, William A Phillips ${ }^{3}$, Joseph T Lizier ${ }^{4}$, Viola Priesemann ${ }^{5,6}$ \\ From 24th Annual Computational Neuroscience Meeting: CNS*2015 \\ Prague, Czech Republic. 18-23 July 2015
}

In many neural systems anatomical motifs are found repeatedly in different places. Despite this repetition these motifs often seem to serve a perplexing variety of functions. A prime example is the canonical microcircuit, which is repeated across multiple cortical areas, but supports a variety of functions from sensory processing and memory to executive functions and motor control. The multiplicity of functions served by a single anatomical motif suggests a common, but more abstract, information processing goal underlying all the different functions. Identifying this goal from neural recordings is a key challenge in understanding the general principles of neural information processing. The apparent diversity of functions makes it clear that this common goal cannot be described using function-specific language (e.g. "edge filters"), but calls for an abstract framework. Here, information theory is the obvious candidate. Notable past approaches using information theoretic descriptions of neural goal functions suggested to maximize the mutual information between input and output [1], maximize the coherent mutual information that all the inputs share about the output [2], or, very generally, to minimize the free energy [3]. To facilitate these efforts, and to better dissect the implications of existing neural goal functions, we suggest to build on a recent progress in information theory, termed partial information decomposition (PID). PID allows to measure which of a set of inputs contributes either uniquely, redundantly or synergistically to the output of a (neural) processing unit [4-7], and which fraction of the output's entropy remains unexplained by the input set. We show how these measures can be used to identify

\footnotetext{
* Correspondence: Wibral@em.uni-frankfurt.de

'MEG Unit, Brain Imaging Center, Goethe University, Frankfurt 60528, Germany

Full list of author information is available at the end of the article
}

an information theoretic footprint of a neural goal function. Most importantly, these measures can quantify how much of the information is modified rather than merely relayed when passing through the neural processor [8]. This shifts the focus from information transmission to more complex processing and allows a much better understanding of the (theoretical?) capabilities of a neuron or neural circuit. Using this approach we show how to better understand existing neural goal functions using PID measures and provide an information theoretic framework for the design of novel goal functions for artificial neural networks.

\section{Authors' details}

'MEG Unit, Brain Imaging Center, Goethe University, Frankfurt 60528, Germany. ${ }^{2}$ Ernst Strüngmann Institute for Neuroscience, Frankfurt 60528, Germany. ${ }^{3}$ School of Natural Sciences, University of Stirling, Stirling FK9 4LA, UK. ${ }^{4}$ School of Civil Engineering, The University of Sydney, Sydney, NSW 2006, Australia. ${ }^{5}$ Department of Nonlinear Dynamics, Max Planck Institute for Dynamics and Self-Organization, 37077 Göttingen, Germany. ${ }^{6}$ Bernstein Center for Computational Neuroscience, 37077 Göttingen, Germany.

Published: 18 December 2015

\section{References}

1. Linsker R: Self-organization in a perceptual network. Computer 1988, 21(3):105-117.

2. Kay JW, Phillips WA: Coherent Infomax as a computational goal for neural systems. Bull Math Biol 2011, 73(2):344-372.

3. Friston K, Kilner J, Harrison L: A free energy principle for the brain. J Physiol Paris 2006, 100(1-3):70-87.

4. Williams PL, Beer RD: Nonnegative Decomposition of Multivariate Information. ArXiv10042515 Math-Ph Physicsphysics Q-Bio 2010.

5. Bertschinger N, Rauh J, Olbrich E, Jost J, Ay N: Quantifying Unique Information. Entropy 2014, 16(4):2161-2183.

6. Griffith V, Koch C: Quantifying Synergistic Mutual Information. In Guided Self-Organization: Inception. Springer Berlin Heidelberg;Prokopenko M 2014:159-190, [Emergence, Complexity and Computation, vol. 9].

7. Wibral M, Lizier JT, Priesemann V: Bits from Brains for Biologically-Inspired Computing. Frontiers in Robotics and Al 2015. 
8. Lizier JT, Flecker B, Williams PL: Towards a synergy-based approach to measuring information modification. Artificial Life (ALIFE), 2013 IEEE Symposium on. IEEE 2013, S43-S51.

doi:10.1186/1471-2202-16-S1-P199

Cite this article as: Wibral et al:: Partial information decomposition as a

unified approach to the characterization and design of neural goal

functions. BMC Neuroscience 2015 16(Suppl 1):P199.

Submit your next manuscript to BioMed Central and take full advantage of:

- Convenient online submission

- Thorough peer review

- No space constraints or color figure charges

- Immediate publication on acceptance

- Inclusion in PubMed, CAS, Scopus and Google Scholar

- Research which is freely available for redistribution

Submit your manuscript at www.biomedcentral.com/submit 